\title{
Social media impact in the Match: A survey of current trends in the United States
}

\author{
Thomas N. Steele, Laura Galarza-Paez, Gabriela Aguilo-Seara, Lisa R. David \\ Department of Plastic and Reconstructive Surgery, Wake Forest School of Medicine, Winston-Salem, NC, USA
}

\begin{abstract}
Background Applicants to integrated plastic and reconstructive surgery (PRS) residency in the United States spend exorbitant amounts of time and money throughout the interview process. Outside of first-hand experience through a visiting rotation, applicants utilize various resources in learning about a program. Today's applicants are "Millennials," the demographic cohort raised during the information age and proficient with digital technology. The authors evaluated whether programs have a presence on social media, and whether applicants are following these accounts.

Methods An online survey was sent to applicants to a single integrated plastic surgery program evaluating basic demographics, social media utilization, and sources of information accessed throughout the residency application process. A manual search of popular social media platforms (Instagram, Facebook, and Twitter) was performed in October 2019. Accounts affiliated with integrated PRS programs were identified and analyzed.

Results Eighty-four of 222 applicants (37.8\%) completed the survey. Ninety-six percent of applicants were within the Millennial demographic. Ninety-six percent of applicants had some form of social media presence, with Facebook (90\%) and Instagram (87\%) being the most popular platforms. Seventy-three percent of applicants reported following a PRS residency social media account. As of October 2019, 59 integrated residency programs (73\%) have active Instagram accounts.

Conclusions Applicants still rely on the program website when researching potential residencies, but social media is being rapidly adopted by programs. Program social media accounts should be used as a dynamic form of communication to better inform applicants of program strengths and weaknesses.
\end{abstract}

Keywords Surgery, plastic / Social media / Internship and residency / Education, medical, graduate
Correspondence: Lisa R. David Department of Plastic and Reconstructive Surgery, Wake Forest School of Medicine, 1 Medical Center Blvd, Winston-Salem, NC 27157, USA

Tel: $+1-318-729-0600$

Fax: +1-336-716-8759

E-mail: Idavid@wakehealth.edu

Received: May 8, $2020 \bullet$ Revised: June 22, $2020 \bullet$ Accepted: October 13, 2020

pISSN: 2234-6163 • elSSN: 2234-6171 • https://doi.org/10.5999/aps.2020.00836• Arch Plast Surg 2021;48:107-113

\section{INTRODUCTION}

Since the creation of the modern residency program by Osler and Halsted in the late 19th century, the process of applying to and being accepted into a residency of choice has grown more complex and competitive. Today, integrated plastic and recon- structive surgery (PRS) is one of the most competitive residencies to which medical students apply. Applicants to PRS residencies frequently rank among the highest United States Medical Licensing Examination (USMLE) scores, volume of research experiences, and percent of Alpha Omega Alpha Honor Society (AOA) membership [1]. 
The recent trend towards adoption of the integrated residency pathway by PRS programs has been coupled with a correlated increase in the Match rate. In the 2019 National Resident Matching Program (NRMP, the Match), there were 78 integrated PRS programs offering 172 positions. In 158 U.S. applicants matched into these positions for a match rate of $92 \%$. In the 2018 Match, this rate was 93\% [2].

Despite the increasing match rate, applicants to integrated PRS spend exorbitant amounts of personal time and finances throughout the pre-interview and interview process [3]. Applicants frequently take a "shotgun" approach, applying indiscriminately to every available program. According to recent literature, applicants in the 2017 Match cycle applied to a median 70 out of 73 programs [4]. Anecdotally, most applicants explain this behavior as resulting from a fear of not matching into a competitive field.

However, this behavior is not without consequence. Molina Burbano et al. [4] discussed the "congestion" that exists in the residency interview process as a result of this indiscriminate application approach. The authors reference the game theory phenomenon of "prisoner's dilemma," previously described in urology residency application by Weissbart et al. [5], which explains why people tend to act in a self-serving manner, even though cooperation amongst individuals would produce a more beneficial result for all.

In order to develop methods to influence applicant behavior, one must first determine what factors guide their behavior. Specifically, how do applicants learn about a residency program? The best exposure to a program is an intimate, first-hand experience. Outside of the home institution, this experience occurs through Acting Internships (AI), also known as sub-internships or away rotations. These rotations can be costly, both in a financial sense and in opportunity cost, but recent studies have shown that program directors consider an applicant's performance on the $\mathrm{AI}$ as one of the most important factors when formulating a rank list [6].

Due to time and financial limitations, most applicants are only able to attend two to four AIs. Outside of these experiences, multiple studies have shown that a program's website is an applicant's primary source of information $[7,8]$. However, program websites are notoriously lacking regarding the information that applicants desire. In 2015, Hashmi et al. [8] reviewed 67 program websites in search of 31 criteria that were important to applicants. They found that only $25 \%$ of program websites had two-thirds of the listed criteria available.

With the rapid evolution of the social media "revolution," all aspects of society are adopting more modern forms of communication, including Instagram, Facebook, and Twitter. Plastic surgeons in particular are at the forefront of utilizing social me- dia, and the influence of social media on the field of PRS has been heavily studied [9-14]. A large portion of Instagram's user base are younger Americans. As of June 2019, 59\% of U.S. Millennials (the generation born between 1981 and 1996) are active on Instagram, and users under 35 years of age make up $70 \%$ of all accounts [15].

Similarly, the demographics of the medical school graduating classes are evolving. Current medical school graduates are now firmly within this "Millennial" generation. Do members of this new generation continue to rely only on websites for sources of information, or is social media a current or potential avenue of communication for programs and applicants to connect?

The aim of this study is to answer two questions: First, in the changing demographics of the PRS residency applicant, what are an applicant's primary sources of information when learning about a program? Second, are programs recognizing an increased demand for modern forms of mass media by increasing their presence on social media platforms?

\section{METHODS}

\section{Survey}

First, a list of fourth-year medical students who applied to a Wake Forest Department of Plastic and Reconstructive Surgery via the Electronic Residency Application Service (ERAS) during the 2018 application cycle was generated. A web-based survey (SurveyMonkey Inc., San Mateo, CA, USA) was created to quantify applicant demographics, social media presence, social media utilization, and what sources of information were accessed by the applicant when researching a PRS residency. The survey consisted of 10 questions and was sent via email (Supplemental Material 1) Completion of the survey was incentivized by rewarding a random participant with a \$25 Amazon gift card.

\section{Social media data}

A list of integrated PRS residency programs participating in the 2018 Match cycle was generated through a downloaded list via the ERAS website. Subsequently, a manual search was performed on three major social media platforms: Instagram, Twitter, and Facebook. A search was conducted using various combinations of the following keywords: plastic surgeon, plastic surgery; reconstructive surgery; residency; plastic surgery residents; resident life; microsurgery; breast reconstruction; and cosmetic surgery. PRS residency program accounts were identified and analyzed. Factors of interest included the date of first post, total number of followers, and total number of posts. All data recorded in this study occurred on a single day-October 9, 2019.

Basic statistical analysis was performed via Microsoft Excel 
(Microsoft Corp., Redmond, WA, USA) to quantify the results of residency program social media data. Analysis included mean, median, range and percentile. Pearson correlation coefficient $\left(\mathrm{r}^{2}\right)$ was calculated in Microsoft Excel to determine correlation between various factors of social media accounts (account age, number of posts, number of followers).

\section{RESULTS}

The total number of applicants to our institution for the 2018 cycle was 222. Eighty-four survey recipients completed the survey for a completion rate of $37.8 \%$. Eighty-one respondents (96\%) are between 23 to 32 years of age. These are firmly members of the "Millennial" generation born between 1986 and 1995. Unsurprisingly, 81 respondents (96\%) endorsed having at least one social media account (Table 1).

The two most popular platforms amongst applicants are Facebook (90\%) and Instagram (87\%). Regarding social media usage, 39 of 84 respondents ( $46 \%$ ) spent one hour or more per day on social media platforms (Table 2). Sixty-one respondents (73\%) reported following a PRS residency social media account.

Table 1. Demographics

\begin{tabular}{lc}
\hline Variable & No. (\%) \\
\hline Age group (yr) & \\
$23-27$ & $48(57)$ \\
$28-32$ & $32(39)$ \\
$33-37$ & $3(4)$ \\
$38-42$ & $1(1)$ \\
Do you have a social media account? & \\
Yes & $81(96)$ \\
No & $3(4)$ \\
\hline
\end{tabular}

Of those respondents that do follow a PRS residency social media account, $92 \%$ followed the programs on Instagram, compared to only 21\% for Facebook and 15\% for Twitter (Table 3).

Applicants were asked what sources of information are utilized when progressing through the residency application process, including sources used when applying for AIs and sources used

\section{Table 2. Social media activity}

\begin{tabular}{lc}
\hline Social media & No. (\%) \\
\hline Presence on social media platform & \\
Facebook & $75(90)$ \\
Instagram & $72(86)$ \\
Twitter & $25(30)$ \\
Snapchat & $63(76)$ \\
Linkedln & $49(59)$ \\
Other & $5(6)$ \\
Time spent on social media (per day) & \\
0-30 minutes & $21(25)$ \\
$30-60$ minutes & $24(29)$ \\
1-2 hours & $24(29)$ \\
2-3 hours & $13(15)$ \\
$3-4$ hours & $1(1)$ \\
$>4$ hours & $1(1)$ \\
\hline
\end{tabular}

Table 3. Social media and program presence

\begin{tabular}{lr}
\hline & No. (\%) \\
\hline Follow PRS residency SM account? & \\
Yes & $61(73)$ \\
No & $23(27)$ \\
SM platforms on which you follow PRS residency accounts & \\
Facebook & $13(21)$ \\
Instagram & $57(92)$ \\
Twitter & $9(15)$ \\
\hline
\end{tabular}

PRS, plastic and reconstructive surgery; SM, social media.

Fig. 1. Applicant survey responses sources of program information

Survey results quantifying where applicants access information when researching programs during away rotations (VSAS) versus interview applications (ERAS). VSAS, Visiting Student Application Service; ERAS, Electronic Residency Application Service.

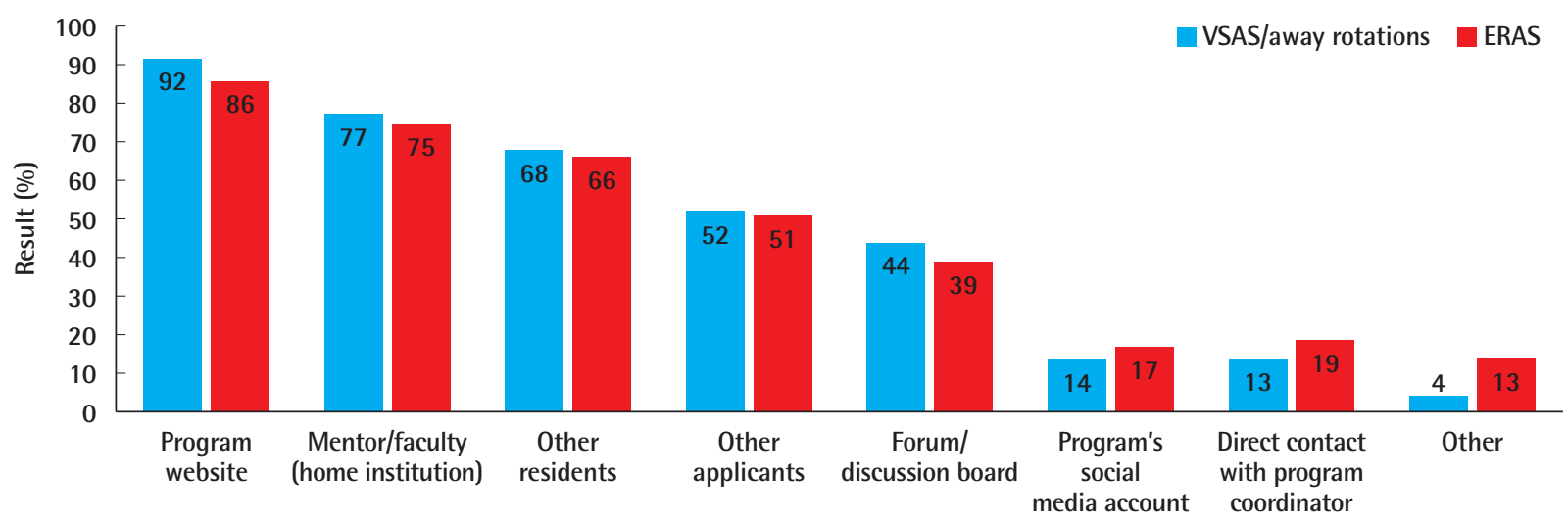


when formally applying for residency interviews through ERAS (Fig. 1). When asked specifically about preparing for the subinternship/away rotation process, 77 respondents (92\%) said that they accessed the website for information, while 71 (86\%) accessed the website in preparation for ERAS.

Regarding interpersonal resources (mentor/faculty members, residents, other students) 64 respondents (77\%) discussed program selection with a mentor or faculty member at their home institution in preparation for applying as an AI, and 62 (75\%) when applying for ERAS. Sixty-eight percent discussed AIs with other residents, either at their home institution or at other institutions, and 66\% did the same during ERAS preparation. Fiftytwo percent and $51 \%$ of respondents discussed AIs and ERAS (respectively) with other applicants. Only 12 respondents (14\%) said they accessed a program's social media account for information during the sub-internship process, and 14 (17\%) during ERAS.

Respondents were then asked how likely they would be to access various sources of information during the residency interview process, given that all choices were available to them (Fig. 2). Nearly all respondents (98\%) were very likely or somewhat likely to access the program website. This was true also for a mentor/faculty at home institution (99\%), other residents (95\%), and other applicants (85\%). 67\% would be very likely or somewhat likely to access a program's social media account, and $65 \%$ would be very likely or somewhat likely to access an online forum/discussion board. Applicants were least likely to utilize direct contact with program coordinator, with $42 \%$ choosing "not likely" or "would not access" this source of information.

Lastly, applicants were asked what information they desired from PRS residency programs' social media accounts. Eightynine percent and $98 \%$ of respondents selected educational content and resident life, respectively. Ten percent selected promotional content. Of the eight free-text responses that selected "Other," the vast majority mentioned research-related content (current areas of research, awards, etc).

The second aspect of this study focused on the social media accounts of PRS residency programs. Fifty-nine integrated programs (73\%) have active Instagram accounts, compared to 23 Twitter accounts (28\%) and 16 Facebook accounts (20\%). Of the 81 integrated programs, 19 (23\%) had no form of social media account through either Facebook, Instagram, or Twitter. Eleven PRS accounts were identified on Instagram that were not associated with an integrated residency program. These were classified as either independent PRS programs, fellowships, or non-U.S. PRS residency programs, and were excluded from analysis.

The earliest identified PRS social media account was on Twitter in October 2009. The earliest Facebook account was December 2010, and the earliest Instagram account was October 2015. Despite Instagram's shorter lifespan, 58 out of 80 programs currently have an active social media account. This is significantly higher than the number of active Facebook (23) and Twitter (16) accounts. The rate of adoption of Instagram by PRS residency programs has increased exponentially, from five active accounts at the end of 2016 to currently 58 unique programs having an active account as of October 9th, 2019 (Fig. 3).

Analysis was performed regarding the age of a PRS residency account, the number of followers, and the number of posts by an account. Regarding Instagram, the number of followers most

\section{Fig. 2. Likelihood of applicants accessing information sources}

Self-reported results of whether an applicant would be likely to access a given source of information.

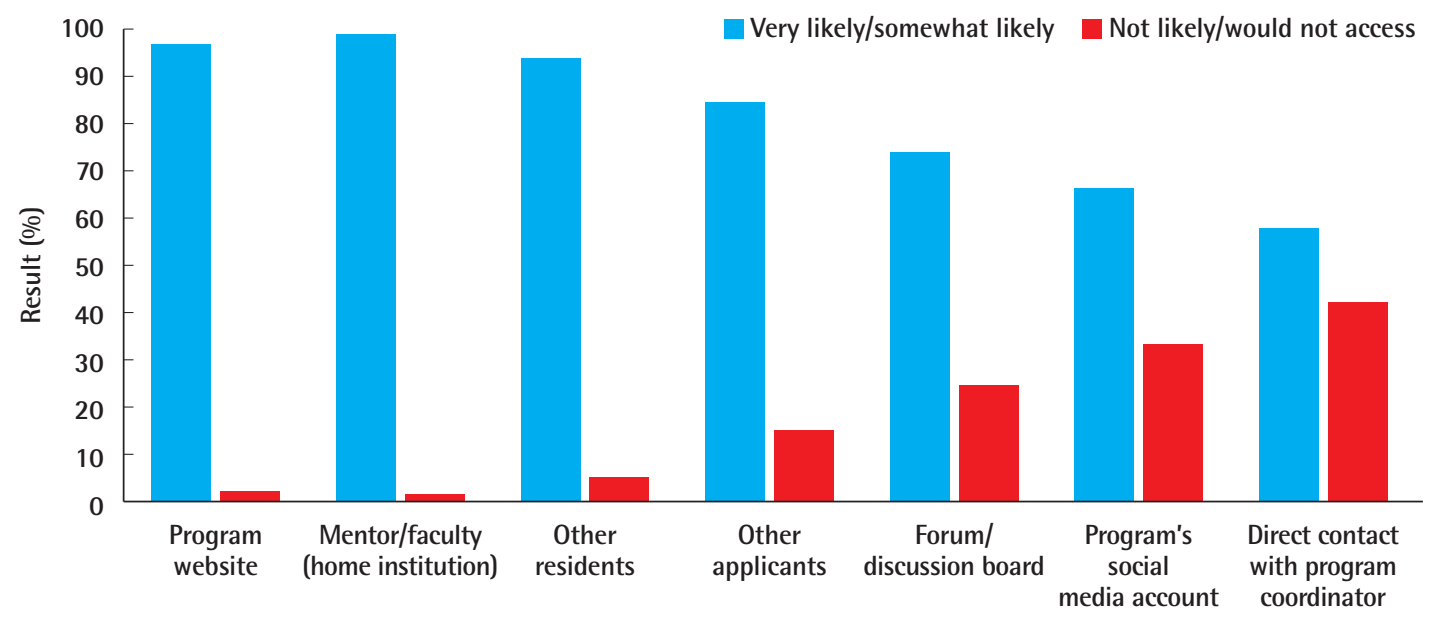




\section{Fig. 3. Plastic surgery residency program social media accounts}

Number of active plastic and reconstructive surgery residency Instagram accounts, cumulative.

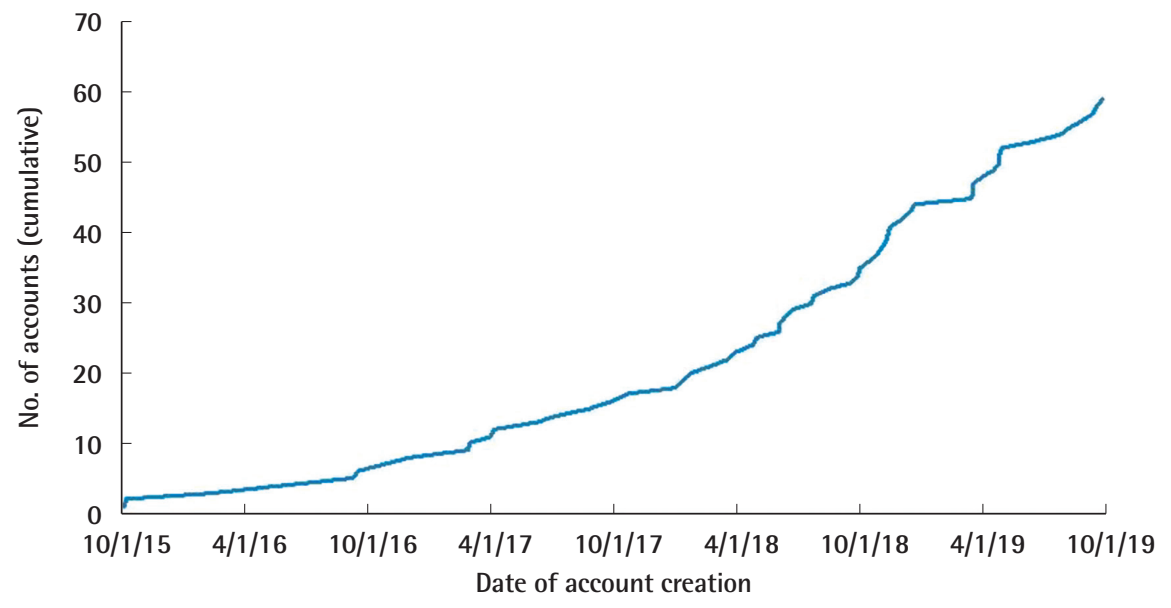

\section{Fig. 4. Analysis of PRS residency social media accounts}

A graphic depiction of comparison between different variables of social media accounts for plastic and reconstructive surgery (PRS) accounts. (A) Number of posts compared to number of followers; (B) number of posts compared to age of account; $(\mathrm{C})$ age of posts compared to number of followers.
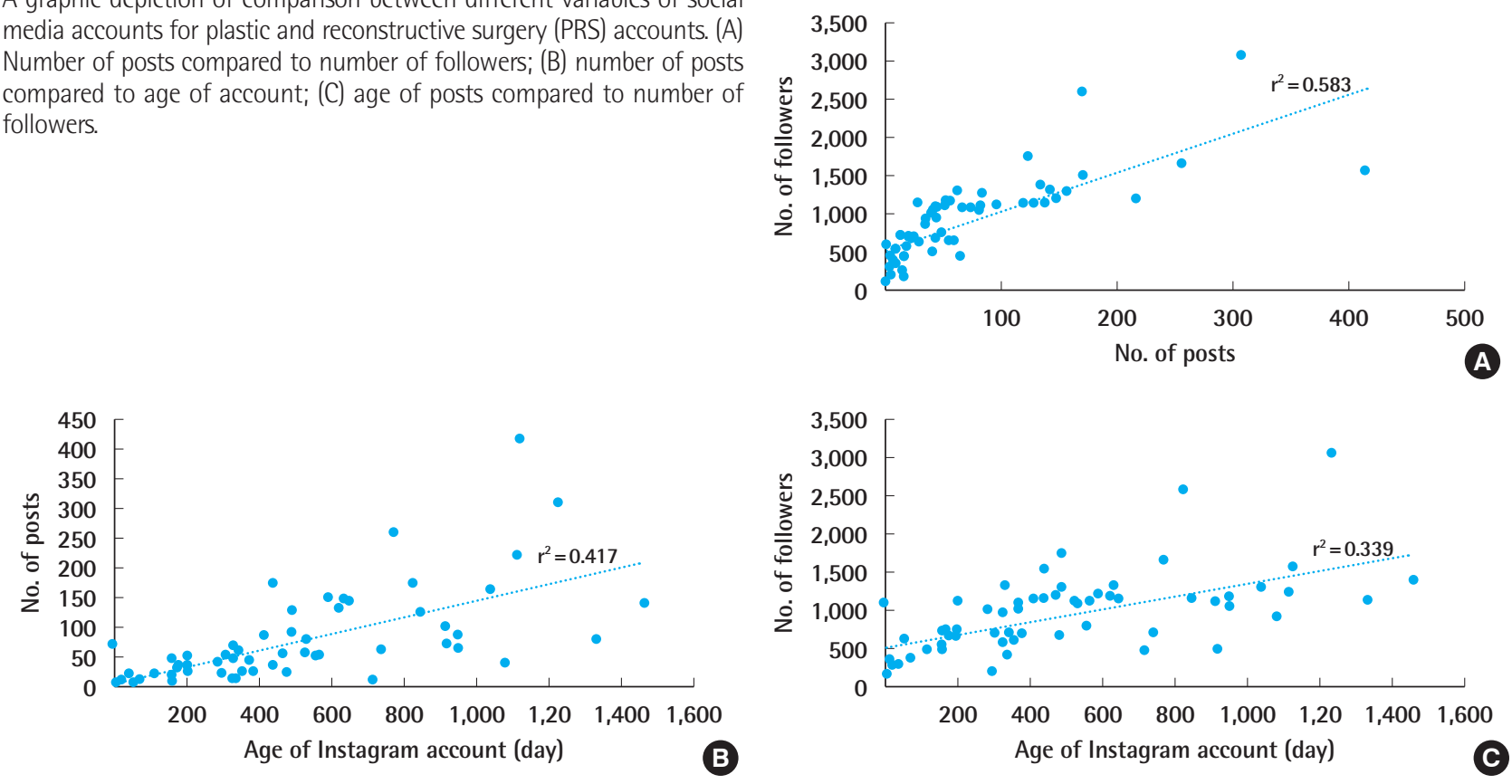

strongly correlated with the number of posts made by an accoefficient of determination $\left(\mathrm{r}^{2}\right)$ surpassing 0.29 . count $\left(r^{2}=0.58\right)$ (Fig. 4A). The age of the Instagram account correlated modestly with number of posts $\left(r^{2}=0.42\right)$ (Fig. 4B), and the age of account compared to the number of followers demonstrated the least correlation $\left(\mathrm{r}^{2}=0.34\right)$ (Fig. 4C).

Due to inability to obtain the number of posts on a program's Facebook account, only age of account and number of followers was obtained, which showed a strong correlation $\left(\mathrm{r}^{2}=0.57\right)$. On Twitter, there was minimal to no correlation between the number of followers, number of posts, and age of account, with no

\section{DISCUSSION}

To the authors' knowledge, this is the first study that has quantified the utilization of resources by applicants to PRS integrated residency programs. Despite the changing applicant demographics, the PRS program website is still the most utilized source of information. Interestingly, interpersonal sources (mentors/faculty, residents, and other students) are a close sec- 
ond source of information. All three of these interpersonal sources are utilized very frequently and are likely valued very highly, given the responses to this survey. However, an individual's opinion of a program is something not easily modified by programs.

When examining the social media trends of the applicant pool, $96 \%$ are in the Millennial generation and, predictably, $96 \%$ also have social media accounts. Presence on Instagram and Facebook were nearly equal- $87 \%$ and $90 \%$, respectively. Interestingly, $73 \%$ of applicants are already following a PRS residency account, and the vast majority of this interaction (92\%) occurs on Instagram, compared to $20 \%$ on Facebook and $15 \%$ on Twitter.

There is some discrepancy between applicants' current behavior and their reported future behavior. Nearly three-quarters of applicants follow a PRS residency social media account, but in 2018 less than $20 \%$ of respondents accessed a program's social media account for information during the interview cycle. However, $67 \%$ said they would be very likely or somewhat likely to access a program's social media account to obtain information during the interview cycle.

It is difficult to tease out the cause of this discrepancy. Perhaps applicants simply don't place much value in a program's social media account. Perhaps programs are on social media but are not active enough to influence their behavior. Or perhaps the impact is subtle or subconscious. Regardless of the cause, there is an opportunity for programs to connect with applicants, both current and future, through social media platforms. And, at least for now, that interaction is most likely to happen on Instagram. Programs should focus their content on resident life and educational content, and avoid posting promotional marketing. In the author's opinion, the PRS residency social media account should be used as a recruiting tool for medical students, not as a tool to recruit patients.

This study also demonstrates that a vast majority of programs have adopted Instagram as a social media platform. The first adopters were in October 2015, almost exactly 5 years after the initial launch of the photo-sharing platform. Whether programs have independently recognized the importance of this new form of communication, or whether matriculating residents have tapped into their familiarity with social media to promote their respective programs, the exponentially increasing presence of programs on Instagram is unmistakable (Fig. 3). But how does our field utilize this knowledge to modify harmful applicant behavior, specifically in the Match process?

Optimizing the Match process is not an easy problem to solve. As discussed earlier, a vast majority of current applicants apply indiscriminately, likely due to a fear of "missing out" or not matching into an integrated position. However, this creates con- gestion in the Match process, which negatively impacts both applicants and programs [4]. Decreased review time of applicants by faculty, institution of "cutoffs" (USMLE, research experiences, AOA status), and overbooking of applicants are all results of this congestion [5]. The indiscriminate application to programs of low interest could preclude an interview opportunity for a more suitable applicant who would be a better mutual fit. If programs are better able to communicate with applicants and broadcast their residency experience and expectations, applicants should theoretically begin selectively applying only to programs that fit their future career goals.

Social media may also be used to reach out to potential applicants who have limited or no exposure to Plastic \& Reconstructive Surgery. There are currently 81 accredited integrated PRS residency programs, but there are 141 M.D. programs and 34 D.O. programs in the United States. Less than half of US medical schools have an affiliated integrated plastic surgery residency program. Medical students at schools without a home institution have a difficult time gaining exposure to the field of PRS, and social media could be an avenue to introduce a broader population of medical students to our field [16].

As discussed earlier, applicants are frequently dissatisfied with the content of information they are able to access on these websites $[7,8]$. This information has already led to changes on a national level. At the 2019 Spring Retreat of the American Council of Academic Plastic Surgeons (ACAPS), a motion was adopted to improve the information found on program websites. Our institution developed a list of criteria that we recommend be easily accessible on all PRS residency websites. These criteria include: program coordinator; program leadership; faculty members; program mission statement; program benefits (parking, on-call meals, room/board stipend, etc); curriculum overview; research expectations; and Information on applying to the program.

This study is not without limitations. First, the response rate of fourth-year medical students is low at 38\%. However, the fact that $96 \%$ of respondents were within the generation of interest lends credibility that this sample is indicative of the applicant pool at large. This rate is also consistent with physician response rates to online polls [17]. Secondly, in the rapidly changing environment of social media popularity, by the time this data is reviewed, a different platform may become the new standard. For example, TikTok is a short-form video-sharing platform that is one of this year's most popular apps for teenagers [18]. This study does not claim to predict the future, but rather provides a snapshot of the present social media climate as it relates to PRS integrated residency applicants and programs. 


\section{NOTES}

\section{Conflict of interest}

No potential conflict of interest relevant to this article was reported.

\section{Author contribution}

Conceptualization: all authors. Data curation: TN Steele, L Galarza-Paez, G Aguilo-Seara. Formal analysis: TN Steele. Methodology: TN Steele, L Galarza-Paez, G Aguilo-Seara. Project administration: TN Steele, LR David. Writing - original draft: TN Steele. Writing - review \& editing: TN Steele, LR David.

\section{ORCID}

Thomas N. Steele

https://orcid.org/0000-0002-7502-4061

Laura Galarza-Paez https://orcid.org/0000-0002-7430-3210

Gabriela Aguilo-Seara https://orcid.org/0000-0002-5673-5001

Lisa R. David https://orcid.org/0000-0003-1233-6479

\section{Supplementary material}

Supplemental Material 1. Applicant survey questions. Supplemental data can be found at: https://doi.org/10.5999/aps.2020. 00836.

\section{REFERENCES}

1. Wood JS, David LR. Outcome analysis of factors impacting the plastic surgery match. Ann Plast Surg 2010;64:770-4.

2. National Resident Matching Program (NRMP). Match Data Archives [Internet]. Washington, DC: NRMP; c2019 [cited 2021 Jan 6]. Available from: http://www.nrmp.org/ category/match-data/.

3. Claiborne JR, Crantford JC, Swett KR, et al. The plastic surgery match: predicting success and improving the process. Ann Plast Surg 2013;70:698-703.

4. Molina Burbano F, Yao A, Burish N, et al. Solving congestion in the plastic surgery match: a game theory analysis. Plast Reconstr Surg 2019; 143:634-9.

5. Weissbart SJ, Hall SJ, Fultz BR, et al. The urology match as a prisoner's dilemma: a game theory perspective. Urology 2013;82:791-7.

6. Janis JE, Hatef DA. Resident selection protocols in plastic surgery: a national survey of plastic surgery program directors. Plast Reconstr Surg 2008; 122:1929-39.

7. Chen VW, Hoang D, Garner W. Do websites provide what applicants need? Plastic surgery residency program websites versus applicant self-reported needs. Plast Reconstr Surg Glob Open 2018;6:e1900.

8. Hashmi A, Policherla R, Campbell H, et al. How informative are the plastic surgery residency websites to prospective applicants? J Surg Educ 2017;74:74-8.

9. Chandawarkar AA, Gould DJ, Stevens WG. Insta-grated plastic surgery residencies: the rise of social media use by trainees and responsible guidelines for use. Aesthet Surg J 2018;38:1145-52.

10. Boczar D, Sisti A, Restrepo DJ, et al. Meaningful use of social media for prospective plastic surgery residency candidates. Plast Reconstr Surg 2019;143:670e-671e.

11. Fan KL, Graziano F, Economides JM, et al. The public's preferences on plastic surgery social media engagement and professionalism: demystifying the impact of demographics. Plast Reconstr Surg 2019;143:619-30.

12. Sorice SC, Li AY, Gilstrap J, et al. Social media and the plastic surgery patient. Plast Reconstr Surg 2017;140:1047-56.

13. Montemurro P, Porcnik A, Heden P, et al. The influence of social media and easily accessible online information on the aesthetic plastic surgery practice: literature review and our own experience. Aesthetic Plast Surg 2015;39:270-7.

14. Rohrich RJ. So, do you want to be facebook friends? How social media have changed plastic surgery and medicine forever. Plast Reconstr Surg 2017;139:1021-6.

15. Perrin A, Anderson M. Share of U.S. adults using social media, including Facebook, is mostly unchanged since 2018 [Internet]. Washington, DC: Pew Research Center; c2019 [cited 2021 Jan 6]. Available from: https://www.pewresearch.org/ fact-tank/2019/04/10/share-of-u-s-adults-using-socialmedia-including-facebook-is-mostly-unchanged-since-2018/.

16. Weber L, Khosravani N. Connecting with the next generation: a medical student's perspective on social media use and plastic surgery. Plast Reconstr Surg 2018;142:247e$248 \mathrm{e}$.

17. Cunningham CT, Quan H, Hemmelgarn B, et al. Exploring physician specialist response rates to web-based surveys. BMC Med Res Methodol 2015;15:32.

18. Leskin P. Inside the rise of TikTok, the viral video-sharing app wildly popular with teens and loathed by the Trump administration [Internet]. New York, NY: Business Insider; c2020 [cited 2021 Jan 6]. Available from: https://www. businessinsider.com/tiktok-app-online-website-video-sharing-2019-7. 


\section{Supplemental Material 1. Applicant survey questions}
1. Age
- 18-22
○ $23-27$
○ 28-32
○ $33-37$
o $38-42$
○ $42+$

2. Do you have a social media account?

- Yes

- No

3. Please select all platforms on which you have an account:
○ Facebook
Instagram
○ Twitter
- Snapchat
○ LinkedIn
o Other

4. How much time (on average) do you spend on social media throughout a 24-hour period?
○ 0-30 minutes
○ 30-60 minutes
○ 1-2 hours
○ 2-3 hours
○ 3-4 hours
○ $>4$ hours

5. Do you follow any Plastic \& Reconstructive Residency social media accounts? Yes/No

6. If "Yes" on Question 5, what platforms do you follow the accounts on?
○ Facebook
Instagram
○ Twitter

7. When selecting which residency programs to apply for away rotations/visiting rotations/sub-internships, what sources of information did you access?

- Program Website

- Online Forum/Discussion Board

- Program's Social Media Account

- Direct Contact with Program Coordinator

- Mentor/Faculty at Home Institution

○ Other Residents

- Other Applicants

○ Other 
8. When selecting residency programs to apply via ERAS, what sources of information did you access?

- Program Website

○ Online Forum/Discussion Board

- Program's Social Media Account

- Direct Contact with Program Coordinator

- Mentor/Faculty at Home Institution

- Other Residents

○ Other Applicants

O Other

9. If all sources listed below were available to you, how likely would you be to access each source in order to gather information about a given residency program? (Very Likely, Somewhat Likely, Not Likely, I would not access this source of information)

- Program Website

○ Online Forum/Discussion Board

- Program's Social Media Account

- Direct Contact with Program Coordinator

- Mentor/Faculty at Home Institution

○ Other Residents

- Other Applicants

10. What type of information would you like to see on a residency program's social media account?

○ Educational content

○ Resident Life

- Promotional Marketing

o Other 